\title{
Smartphone App to Deliver Virtual Follow-up Care for Children with Type 1 Diabetes During the COVID-19 Pandemic: Experience in a Resource-Limited Setup
}

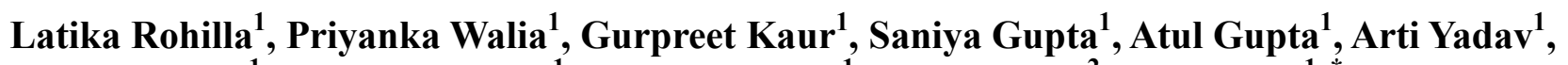 \\ Pamali Nanda ${ }^{1}$, Jaivinder Yadav ${ }^{1}$, Rakesh Kumar ${ }^{1}$, Nancy Sahni ${ }^{2}$, Devi Dayal ${ }^{1, *}$ \\ ${ }^{1}$ Department of Pediatrics, Postgraduate Institute of Medical Education and Research, Chandigarh, India \\ ${ }^{2}$ Department of Dietetics, Postgraduate Institute of Medical Education and Research, Chandigarh, India
}

Email address:

drdevidayal@gmail.com (D. Dayal)

${ }^{*}$ Corresponding author

\section{To cite this article:}

Latika Rohilla, Priyanka Walia, Gurpreet Kaur, Saniya Gupta, Atul Gupta, Arti Yadav, Pamali Nanda, Jaivinder Yadav, Rakesh Kumar, Nancy Sahni, Devi Dayal. Smartphone App to Deliver Virtual Follow-up Care for Children with Type 1 Diabetes During the COVID-19 Pandemic: Experience in a Resource-Limited Setup. International Journal of Diabetes and Endocrinology. Vol. 6, No. 2, 2021, pp. 64-68. doi: $10.11648 /$ j.ijde.20210602.12

Received: April 5, 2021; Accepted: April 14, 2021; Published: April 26, 2021

\begin{abstract}
COVID-19 pandemic has forced the medical fraternity to quickly adopt telemedicine for patient care. However, the feasibility and utility of smartphone app-based telecare for children with type 1 diabetes (T1D) in resource-limited settings is scarcely reported. A web-based survey, developed with "Google forms", was sent to parents via the smartphone app WhatsApp, and the responses to the 12 coded questionnaire items were analyzed. The multiple-choice questions were related to the experiences, satisfaction, and expectations regarding the follow-up care delivered to children with T1D between April and November 2020. A total of 578 queries were resolved through WhatsApp during the study period. These were mostly related to reviewing reports, insulin titration, or other minor ailments. From 332 participants in the two WhatsApp groups, 183 responses $(55.1 \%)$ were collected. The majority of respondents found the WhatsApp telecare model as easy to learn (69.4), simple to use $(68.3 \%)$ and were overall satisfied $(64.5 \%)$. None of the families reported that they would recommend against the use of telecare through WhatsApp. Most of them (45.9\%) felt that this model could be used in addition to their physical follow-up visits. However, only $15.3 \%$ felt that this model could replace all clinic visits in the future. Only $12 \%$ of the respondents reported technical difficulties. In conclusion, virtual follow-up care delivered through WhatsApp is feasible and acceptable to families of children with T1D. The minor challenges related to internet access and low literacy levels were easily overcome. WhatsApp-based virtual visits may complement physical visits to the clinic, especially for patients from remote areas.
\end{abstract}

Keywords: COVID-19 Pandemic, Telemedicine, WhatsApp, Type 1 Diabetes, Follow-up Visits, Children

\section{Introduction}

The coronavirus 2019 (COVID-19) pandemic has severely disrupted healthcare services throughout the world. The redistribution of healthcare resources to COVID-19 care and severe lockdown restrictions made healthcare access difficult for non-COVID-19 conditions, especially for patients suffering from chronic diseases that require periodic assessment and changes in treatment plans. The medical fraternity quickly adopted alternative models of delivery of medical care for patients suffering from non-COVID-19 chronic conditions [1]. Of the several digital approaches, smartphone applications and instant messaging services are being commonly employed for communication between medical teams and patients [1]. One such communication tool, the smartphone app WhatsApp, allows users to send instant messages, photos, videos, and voice messages. It can also be used to share medical records and make voice or video calls using a mobile internet connection. Besides, the possibility to create protected groups, the so-called 
WhatsApp groups make WhatsApp useful for cooperative teleconsultation [2]. WhatsApp has thus emerged as the primary telemedicine tool for virtual care during the ongoing pandemic $[1,2]$.

Type 1 diabetes (T1D) is one of the common chronic conditions during childhood and adolescence [3]. The patients require periodic follow-up visits to the clinic after the diagnosis of T1D. The onset of the COVID-19 pandemic caused a severe disruption in the follow-up care of children with T1D, besides causing delays in the diagnoses and acute care $[4,5]$. In addition to several considerations for children and adolescents with T1D, an urgent and rapid expansion of telemedicine services for delivering diabetes care, precisely the virtual follow-up care, was quickly adopted by several institutions across the world during the initial phase of the pandemic $[6,7]$.

Ours is the largest tertiary care pediatric referral hospital that caters to four federal states and two union territories of northwest India [8]. Since the inception of pediatric diabetes services in 2004, all parents of children with T1D are required to provide a working phone number at the time of registration in the outpatient clinic, which is mainly used for follow-up reminders or intimations about diabetes-related educational events. To improve communication between the diabetes team and parents, we created the first WhatsApp group (Diabetes Kids Group 1) in November 2018, which included most children on active follow-up at that time. The use of WhatsApp was, however, restricted to attending to the emergency queries from parents. As the number of participants increased, the second group (Diabetes Kids Group 2) was created in July 2020. The WhatsApp queries are handled by a member of the diabetes team that comprises diabetes nurses, DM fellows, consultant dieticians, and consultant pediatric endocrinologists. Because of the COVID-19 pandemic and the ensuing nationwide lockdown in March 2020, we expanded our WhatsApp service to include all types of patient queries and review of selfmonitoring blood sugar (SMBG) records and laboratory reports. Although we started perceiving the usefulness of WhatsApp communication during the first few weeks of its expanded use, a formal assessment remained pending.

Most of the previous studies on virtual care for children with T1D during the COVID-19 pandemic have been conducted in the developed countries where patients do not face problems such as understanding the WhatsApp communication due to low educational status or poor quality of internet connections and smartphone devices [7, 9, 10]. The data from resource-constrained settings like ours are scarce [11]. We cater to a patient population with low levels of literacy and limited financial resources. Several families do not have computers or Wi-Fi at home. However, almost all families have cell phones with the availability of WhatsApp, which allowed us to use this tool for virtual care during the COVID-19 pandemic. The current study was planned to share our experience on WhatsApp's usefulness to deliver follow-up diabetes care to children with T1D.

\section{Methods}

For assessing the usage of the WhatsApp service, a qualitative content analysis of the WhatsApp conversations from April 12020 to November 30, 2020 was conducted. Codes and themes were generated about the types of queries raised by the group members. We developed a web-based survey using Google forms with multiple-choice questions related to the experiences, satisfaction, and expectations regarding the follow-up care delivered to children with T1D, which was sent to the families via WhatsApp. The assessment was done using a structured tool adapted from the Telehealth usability questionnaire (TUQ) [12]. The questionnaire was prepared in English as well as Hindi. The responses to the coded questionnaire items were analyzed. Responses of the participants $(n=25)$ who expressed difficulty in comprehending survey items were recorded telephonically. Additionally, 50 randomly selected participants from those who had not responded during the two weeks (1 December 2020 to 15 December 2020) of initiating the survey were contacted telephonically, and their responses were recorded if they expressed their willingness to participate voluntarily. The study design was crosssectional.

\section{Results}

During the study period, 578 queries were resolved in the two groups (Table 1). The mean number of queries attended in a month was 73 (range, 36-93). Maximum queries (27.2\%) were related to routine follow-up laboratory investigations such as glycosylated hemoglobin (HbAlc) and lipid profile and reviewing the reports. This was followed by queries concerning insulin titration $(24.4 \%)$ and other minor ailments not directly related to diabetes $(21.9 \%)$. For advice related to insulin titration, parents were required to send a photograph of their SMBG logbook pages for the past few days using their smartphone camera and to upload it to the WhatsApp group. Advice on symptoms of diabetic ketoacidosis (DKA) was required only nine times; none of these children required hospital admission.

The number of participants in the two WhatsApp groups was 332 till 30 November 2020. Of the 195 responses received, 12 duplicates were removed. The remaining 183 survey forms (response rate, 55.6\%) were finally analyzed. The mean age of children was $9.3 \pm 4.4$ years. The duration of diabetes was less than one year, one-two years, and more than two years in $24.1 \%, 42.1 \%$, and $33.9 \%$ patients, respectively. A majority (82\%) of the respondents were parents, $13.7 \%$ were parent and child together, and $4.4 \%$ were the children themselves. The responses about the feasibility and utility are presented in Table 2. Complete satisfaction with the use of WhatsApp communication was expressed by $64.5 \%$ of participants while $69.4 \%, 68.3 \%$, $44.8 \%$, and $63.9 \%$ found this model easy to learn, simple to use, capable of saving traveling time to the hospital, and easy to understand, respectively. For answering queries in the 
future, $64.5 \%$ of respondents felt the need for dieticians, while $55.2 \%, 50.3 \%$, and $48.6 \%$ of respondents wished their

queries to be handled by DM fellows, diabetes nurse educators, and consultants.

Table 1. Type and number of queries resolved through the use of WhatsApp from April to November 2020.

\begin{tabular}{|c|c|c|c|c|c|c|c|c|c|}
\hline Type of query & April & May & June & July & August & September & October & November & Total (\%) \\
\hline Diet-related & 0 & 3 & 2 & 0 & 1 & 4 & 3 & 1 & $14(2.4)$ \\
\hline Insulin titration & 15 & 14 & 14 & 22 & 17 & 30 & 15 & 14 & $141(24.4)$ \\
\hline DKA symptoms & 0 & 2 & 0 & 1 & 4 & 1 & 1 & 0 & $9(1.6)$ \\
\hline Insulin administration & 5 & 2 & 5 & 5 & 3 & 4 & 4 & 2 & $30(5.2)$ \\
\hline SMBG & 0 & 1 & 1 & 1 & 2 & 0 & 3 & 2 & $10(1.7)$ \\
\hline Due tests and reviewing reports & 4 & 12 & 17 & 24 & 30 & 14 & 33 & 23 & $157(27.2)$ \\
\hline Telemedicine & 4 & 4 & 7 & 4 & 3 & 6 & 6 & 10 & $44(7.6)$ \\
\hline Miscellaneous & 3 & 3 & 4 & 10 & 7 & 10 & 6 & 3 & $46(8)$ \\
\hline Total & 36 & 53 & 61 & 93 & 85 & 92 & 90 & 68 & $578(100)$ \\
\hline
\end{tabular}

Abbreviations: DKA, diabetic ketoacidosis; SMBG, self-monitoring blood glucose

Table 2. Questionnaire items and responses by parents and children.

\begin{tabular}{|c|c|c|c|c|}
\hline Item & Not at all & Partly & Quite a bit & Completely \\
\hline Improved access to healthcare services? & $1(0.5)$ & $32(17.5)$ & $76(41.5)$ & $74(40.4)$ \\
\hline Saved time traveling to a hospital? & $2(1.1)$ & $20(10.9)$ & $79(43.2)$ & $82(44.8)$ \\
\hline Provided for the child's healthcare needs? & $1(0.5)$ & $30(16.4)$ & $74(40.4)$ & $78(42.6)$ \\
\hline Using WhatsApp group for queries was easy to learn? & $1(0.5)$ & $15(8.2)$ & $40(21.9)$ & $127(69.4)$ \\
\hline Using WhatsApp group for queries was simple? & $1(0.5)$ & $15(8.2)$ & $42(23)$ & $125(68.3)$ \\
\hline Liked using the WhatsApp group? & $1(0.5)$ & $13(7.1)$ & $51(27.9)$ & $118(64.5)$ \\
\hline WhatsApp group reply was easy to understand? & $1(0.5)$ & $13(7.1)$ & $52(28.4)$ & $117(63.9)$ \\
\hline Could easily interact with staff using the WhatsApp group? & $4(2.2)$ & $18(9.8)$ & $64(35)$ & $97(53)$ \\
\hline Could understand the doctor just as well as if met in person? & $4(2.2)$ & $31(16.9)$ & $70(38.3)$ & $78(42.6)$ \\
\hline $\begin{array}{l}\text { In general (not just during COVID-19), is the WhatsApp group an acceptable way } \\
\text { to receive healthcare services? }\end{array}$ & $5(2.7)$ & $41(22.4)$ & $49(26.8)$ & $88(48.1)$ \\
\hline $\begin{array}{l}\text { In general (not just during COVID-19), would choose to use WhatsApp group } \\
\text { again for interacting with your doctor? }\end{array}$ & $2(1.1)$ & $26(14.2)$ & $50(27.3)$ & $105(57.4)$ \\
\hline
\end{tabular}

Twenty-two (12\%) participants reported difficulty in sharing records. The reasons were difficult internet access in $45.5 \%$, difficulty in clicking or uploading photographs in $13.6 \%$ each, or lack of time in $27.3 \%$. Other problems in using cell phone devices and WhatsApp included low photographic quality of SMBG records and inability to articulate or understand messages due to low education level. These challenges were overcome by voice messages or voice calls and by allowing the use of Hindi language texts. Irrelevant messages needed to be continuously checked and affirmatively stopped to maintain decorum in the group. Occasionally, the needed advice was given after discussion with the consultants; all WhatsApp conversations between a member of the diabetes team and families were supervised by one of the consultants.

\section{Discussion}

Several telemedicine tools have shown potential in the management of diabetes in general and in improving glycemic control precisely [13]. The evidence of their usefulness was, however, confined to the non-crisis situations. With the advent of COVID-19, routine diabetes care had to be delivered through digital platforms due to restrictions on travel and lack of healthcare access. Several advanced diabetes centers in the developed countries that adapted and expanded their telemedicine services have reported benefits of virtual care during the COVID-19 pandemic $[1,2,7,9,10]$. The reported effectiveness of virtual diabetes care in high-income countries is due to better internet services and connectivity, the use of technologically advanced smartphone devices, and the parents' ability to communicate, which largely depends on their higher literacy levels $[9,10]$. However, there are several constraints in using virtual care in the context of developing countries [14]. The acceptance of virtual care advice may be low as patients are conventionally used to physical visits. Internet services and connectivity is poor in remote areas. Additionally, several individuals need help in operating smartphone devices due to their low literacy [14]. Thus, the data on the feasibility and utility of telemedicine for childhood diabetes in resourceconstrained settings remains scarce. A recent study from a lowresource country found that telemedicine care was useful for children with diabetes during the COVID-19 pandemic [11]. Our current study results in a comparable developing country set up are similar and further demonstrate the usefulness of the exclusive use of the smartphone app WhatsApp to deliver virtual follow-up care for children with T1D.

A notable feature of our study was a high level of satisfaction with the use of WhatsApp for follow-up care expressed by almost two-thirds of families. This is consistent with the high satisfaction rates observed in previous similar studies concerning diabetes care during the COVID-19 pandemic [9, 10]. Additionally, almost all families found WhatsApp communication easy to learn and use; this 
indicates that this telemedicine tool is feasible for virtual care in our settings. The finding that approximately $80 \%$ of families felt the WhatsApp care was as good as physical care hints at the possibility of a reduction in the number of inperson follow-up clinic visits in the future. This will be particularly useful for patients with limited financial resources and living in remote areas who find traveling to the hospital costly and time-consuming [14].

The strength of our study is a high response rate comparable to or slightly better than the previous studies [7, 9-11]. The participants represented the overall population of our diabetes clinic. Unlike other telehealth implementing programs, this model did not require training for the end-users or the health care professionals [15]. Furthermore, we did not observe a previously reported limitation of self-selection for participation in the survey, raising the risk of responder bias [9]. This limitation was pre-empted by telephonic interviews of 50 randomly selected participants, which also increased the generalizability of the findings. Another strength of our study is the demonstration of the usefulness of WhatsApp communication in avoiding DKA-related hospital admissions during the COVID-19 pandemic when most hospitals were not accessible for non-COVID care [4-6, 16]. All nine children who showed a risk for DKA were managed remotely by the diabetes team, and further deterioration or need for hospitalization was successfully avoided.

The role of telecare has been expanding in recent times. Although legal and ethical concerns pertaining to confidentiality, data security, dignity, and privacy generally become irrelevant during crisis times, these are still crucial during non-crisis times. We did not encounter any of such issues during our study probably because timely and widely publicized guidelines on telecare were issued by the national government [17]. Future studies regarding virtual diabetes care for children with T1D should capture the health care professionals' perspectives too. The diabetes team members may sometimes feel an extra burden of resolving queries in the WhatsApp group when the hospitals are running busy. The existing workforce may need to be enhanced to take up the additional burden of virtual care [14]. We suggest focused group discussions and in-depth interviews of both the health care professionals and families having children with T1D to generate insights about which families will benefit from telehealth for diabetes management. A head-to-head comparison of in-person and virtual consultations can also pave the way to future virtual or hybrid diabetes care.

\section{Conclusion}

Our experience suggests that virtual diabetes care delivered through the commonly used smartphone app WhatsApp is feasible and useful in a resource-constrained setting. WhatsApp's use for follow-up care of children with T1D was easy, safe, and almost as effective as in-person clinic visits. Minor difficulties related to internet access and low literacy levels of families were easy to circumvent.

\section{Conflict of Interest}

The authors declare that they have no competing interests.

\section{Statement of Ethics}

All relevant ethical guidelines have been followed for data collection and reporting.

\section{References}

[1] Badawy SM, Radovic A. Digital Approaches to Remote Pediatric Health Care Delivery During the COVID-19 Pandemic: Existing Evidence and a Call for Further Research. JMIR Pediatr Parent. 2020; 3: e20049.

[2] Giansanti D. WhatsApp in mHealth: an overview on the potentialities and the opportunities in medical imaging. Mhealth. 2020; 6: 19.

[3] Gupta BM, Dayal D. Pediatric type 1 diabetes research in the 21st century: a scientometric review. Pediatr Endocrinol Diabetes Metab. 2020; 26: 132-139.

[4] Dayal D, Gupta S, Raithatha D, Jayashree M. Missing during COVID-19 lockdown: Children with onset of type 1 diabetes. Acta Paediatr. 2020; 109: 2144-2146.

[5] Dayal D, Gupta S, Raithatha D, Soni P, Kumar S, Baranwal A. Near-fatal experience due to delayed diagnosis of type 1 diabetes during the COVID-19 pandemic. April 28, 2020, Preprint (Version 1). Available at Research Square https://doi.org/:10.21203/rs.3.rs-25337/v1.

[6] Dayal D, Yadav A. COVID-19: Considerations for children and adolescents with diabetes. J Diabetol. 2020; 11: 126-130.

[7] March CA, Flint A, DeArment D, Gilliland A, Kelly K, Rizzitano E, et al. Paediatric diabetes care during the COVID19 pandemic: Lessons learned in scaling up telemedicine services. Endocrinol Diabetes Metab. 2020: e00202.

[8] Dayal D, Saini AG, Jayashree M, Singhi S, Kumar R, Samprati M, Singh M. Hospital based incidence, patterns of presentation and outcome of Type 1 diabetes: 12 years' data from a tertiary care center in North India. Int J Diabetes Dev Ctries. 2015; 35: 103-107.

[9] Fung A, Irvine M, Ayub A, Ziabakhsh S, Amed S, Hursh BE. Evaluation of telephone and virtual visits for routine pediatric diabetes care during the COVID-19 pandemic. J Clin Transl Endocrinol. 2020; 22: 100238.

[10] De Guzman KR, Snoswell CL, Taylor ML, Senanayake B, Haydon HM, Batch JA, et al. A systematic review of pediatric telediabetes service models. Diabetes Technol Ther. 2020; 22: 623-38.

[11] Zabeen B, Bhowmik B, Huda K, Naz F, Tayyeb S, Azad K. Use of Telemedicine for the Management of Type 1 Diabetes in Children and Adolescents in Bangladesh During the COVID-19 Pandemic. J Diabetol. 2021; 12: 18-21.

[12] Parmanto B, Lewis AN Jr, Graham KM, Bertolet MH. Development of the Telehealth Usability Questionnaire (TUQ). Int J Telerehabil. 2016; 8: 3-10. 
[13] Faruque LI, Wiebe N, Ehteshami-Afshar A, Liu Y, DianatiMaleki N, Hemmelgarn BR, et al. Effect of telemedicine on glycated hemoglobin in diabetes: A systematic review and meta-analysis of randomized trials. CMAJ. 2017; 189: E34164.

[14] Banerjee M, Chakraborty S, Pal R. Teleconsultation and Diabetes Care Amid COVID-19 Pandemic in India: Scopes and Challenges. J Diabetes Sci Technol. 2020; 14: 714-715.

[15] Fogel JL, Raymond JK. Implementing Telehealth in Pediatric Type 1 Diabetes Mellitus. Pediatr Clin North Am. 2020; 67: 661-664.
[16] Peters AL, Garg SK. The Silver Lining to COVID-19: Avoiding Diabetic Ketoacidosis Admissions with Telehealth. Diabetes Technol Ther. 2020; 22: 449-453.

[17] Telemedicine Practice Guidelines Enabling Registered Medical Practitioners to Provide Healthcare Using Telemedicine. March 25, 2020, MoHFW, Government of India. Available at: https://www.mohfw.gov.in/pdf/Telemedicine.pdf. Accessed January 8, 2021. 